\title{
Research on the Current Situation and Countermeasures of Higher Mathematics Education
}

\section{Shengliu Li}

Baise University, Baise, Guangxi, 533000

\begin{abstract}
Keywords: higher mathematics education; education status; impact and countermeasures
\end{abstract}
\begin{abstract}
As an important public foundation course, higher mathematics is a scientific language used by many disciplines and plays an important role in the cultivation of talents in higher education institutions. At present, there are still many shortcomings in higher mathematics teaching in colleges and universities. Based on the analysis of the current situation of higher mathematics teaching, this paper puts forward the principles that should be followed in higher mathematics teaching, and further elaborates the reform and basic countermeasures of higher mathematics teaching.
\end{abstract}

\section{Introduction}

In recent years, China's higher education has developed greatly, and a large number of applied talents have been delivered to the society. The combination of various disciplines and higher mathematics will be more extensive and in-depth, and the position of mathematical thinking and quality in the overall quality of talents is receiving more and more attention. However, higher mathematics as a public culture course, a compulsory course for higher education, has not received the attention it deserves in higher education, especially without the attention of students. Failure to take effective measures in a timely manner will affect the quality of higher mathematics teaching and the learning of subsequent professional courses, which is not conducive to the cultivation of innovative talents.

\section{The Status Quo of Higher Mathematics Teaching}

Throughout the present, most of the higher mathematics textbooks are basically deleted on the basis of the original college or adult specialist textbooks, but they are not satisfactory. For example, the differential mean value theorem is an important theorem of calculus in the basic position, including three theorems: Roel theorem, Lagrangian theorem, Cauchy theorem. The Rolle theorem is the basis for proving the other two theorems. The proof process reflects the law of continuous changeable function. In contrast, the latter two theorems are more like a description after the conclusion is found, but the proof is not so important. However, some high-level textbooks have omitted the proof of the Rolle theorem, which is puzzling. In addition, the textbook itself cannot be well integrated with higher vocational professions, without applicability and practicality, lack of mutual penetration with other professional disciplines, and the ability to apply mathematical principles and methods to solve practical problems in the profession is difficult to achieve, lacking higher education. Features. For example, many majors in science and engineering, such as chemical engineering, electromechanical, etc., use the same advanced mathematics textbooks, and the textbook itself is not strong in connection with various majors. Students do not realize the influence of higher mathematics on the profession, and they do not feel its importance.

In order to improve the employment rate, colleges and universities often attach importance to the cultivation of vocational skills, focusing on the construction of professional courses and skills training. The basic and basic mathematics classes are reduced and reduced, and even some majors do not open higher mathematics classes. Due to the lack of class hours, teachers have to keep up with the progress of the class, often full of house irrigation, affecting the quality of teaching. The contradiction between mathematics teaching content and mathematics lesson has obviously brought 
great difficulties to mathematics teaching.

The students in higher education institutions are mainly admitted to the students who have achieved the fourth batch of admission scores in the college entrance examination. Therefore, there are problems such as poor mathematics foundation and lack of mathematics application ability. It is easy to produce difficult mathematics when exposed to more abstract higher mathematics. Even the feelings of being tired of learning, and many other professions are both arts and sciences, which leads to the phenomenon of both liberal arts students and science students in the same class. It is difficult to take care of every student in the teaching process, and teachers and students feel at a loss.

\section{Principles for Higher Mathematics Teaching}

Higher education should advocate the all-round development of people. Paying attention to the cultivation of students' personality, morality and comprehensive quality must follow the principle of people-oriented and student-centered, and truly reflect the cultural and educational functions and subtle influences of higher mathematics courses. Mathematical knowledge comes from social practice and promotes the progress and development of human society. It is a kind of science culture and a commonly used technology. Teachers should help students understand the history, application, development trend of mathematics and the innovation of mathematicians. Spirit, etc., reflects the role of social development in the promotion of mathematical science, clarifies the application of mathematics in various economic and social industries, and the enormous influence of mathematics on the lifelong development of a person, and educates students to form a correct mathematical view. On the basis of paying attention to students' knowledge, ability and value, higher vocational mathematics should pay more attention to the development of students' individuality. It should follow the development line of "knowledge, quality and ability" to deepen the reform of higher vocational mathematics teaching, not only to meet professional development. The need for mathematics curriculum should promote the physical and mental health of students. It is necessary to ensure the quality of students' employment and promote the sustainable development of students. It is necessary to pay attention to the transformation of curriculum teaching from unity to comprehensiveness, overcoming the mathematics curriculum and paying too much attention to knowledge and computing. The tendency to achieve "everyone learns valuable mathematics, everyone can get the necessary mathematics, and different people get different developments in mathematics."

In the reform of higher mathematics teaching in colleges and universities, it is necessary to establish a new concept of education and establish a new concept of curriculum reform. The curriculum should focus on professional services, pointing to the development of students' potential and individuality; the curriculum reform must attach great importance to the liberation of human beings. It is consistent with the professional development of vocational students; it is necessary to highlight the applicability, comprehensiveness and development of the curriculum so that the curriculum truly serves the sustainable development of college students. The curriculum characteristics of modern vocational education are mainly reflected in the curriculum standards. The curriculum standards of higher education institutions are an important basis for textbook writing, teaching design, teaching organization, teaching implementation and curriculum evaluation. They are the basic content of teaching management in higher education institutions. It reflects the characteristics of higher education curriculum, fully reflects the requirements of students and professional needs, rationally formulates the nature, objectives, framework and content system of the curriculum, and proposes curriculum teaching suggestions, evaluation requirements and teaching strategies. Therefore, the standards of higher vocational mathematics curriculum should include: first, the nature and orientation of the curriculum, curriculum design philosophy and curriculum objectives; second, the basic framework of the curriculum, time, key points and difficulties of teaching, teaching methods and means, learning situations, etc., ability requirements and evaluation recommendations The third is the implementation of teaching recommendations, the basic requirements of students in terms of knowledge, skills and emotional attitudes and values, the 
demand for higher mathematics of different majors and the training standards that should be achieved.

The students in higher education institutions are mainly admitted to the students who have achieved the fourth batch of admission scores in the college entrance examination. Therefore, there are problems such as poor mathematics foundation and lack of mathematics application ability. It is easy to produce difficult mathematics when exposed to more abstract higher mathematics. Even the feelings of being tired of learning, and many other professions are both arts and sciences, which leads to the phenomenon of both liberal arts students and science students in the same class. It is difficult to take care of every student in the teaching process, and teachers and students feel at a loss.

\section{Countermeasures for Higher Mathematics Teaching}

It is recommended that all institutions of higher learning, in accordance with their actual situation, organize teachers to prepare teaching materials and handouts suitable for the school and the major. The teachers and departmental teachers will discuss and determine the content of the lectures, and strive to meet the current and future development of the students. This is conducive to the realization of the overall goal, so that students are more targeted when learning.

Higher mathematics is an important part of college teaching, but different professions have different requirements for mathematics. It is unscientific to use the same teaching materials and learn the same content. It is unscientific for both teaching and learning. Mathematical knowledge is applied to the consciousness and ability of the major, and students' emotions and attitudes towards mathematics are cultivated. Some content can be added or selected according to different professions. For example, engineering majors focus on learning tools such as drawing tools, fluid pressure, curvature and radius of curvature, and engineering mathematics; accounting and economic management majors focus on minimum input and maximum benefit, best practices, marginal cost, probability, Learning of statistics and other content. For students who have the ability to learn and are particularly interested in mathematics, they can set up a training course on Mathematical Modeling to enable these students to make full use of their spare time to develop their skills.

At present, the single knowledge structure of higher mathematics teachers in higher education institutions is a common problem. The continuing education of mathematics teachers is not limited to this major, but should be strengthened in education and learning in other professions. For example, teachers who teach advanced mathematics courses in electromechanical majors should receive education and learning in electromechanical expertise to understand and master the necessary professional background knowledge. Only by strengthening the interdisciplinary education of mathematics teachers can we completely solve the problem of single knowledge structure and change the current teaching situation of higher mathematics. Therefore, mathematics teachers only have a certain understanding and mastery of the relevant fields of professional (such as mechanical and electrical, petroleum, chemical and other professional), in order to truly grasp the "necessary, sufficient" degree in future teaching.

In higher vocational mathematics teaching, strict mathematical argumentation should be diluted, emphasizing geometric description, emphasizing intuitive and visual understanding, and freeing students from cumbersome mathematical derivation and general mathematical skills. When explaining advanced mathematics knowledge, it should be based on the study of familiar life examples or examples of professional integration, which fully reflects the students' participation and autonomy. For example, when explaining the concept of derivative, in addition to the speed of shifting linear motion introduced in the problem of rate of change on books, some problems related to rate of change are introduced. For example, in the course of electromechanical teaching, the problem of the rate of change of the density of non-uniformly distributed fine rods, and the current intensity of non-constant currents can be introduced. Only students who have a large number of contacts with the profession to explain mathematics knowledge can enable students to establish real mathematical concepts, improve the overall teaching effect, and at the same time reflect the ideological and service nature of higher mathematics. 
Breaking mathematics is only a problem class, and there is no traditional mode of experiment class. Open mathematics experiment classes to develop students' mathematical modeling ability, scientific calculation methods and means, and data processing capabilities. To enable students to understand the perfect combination of mathematics and modern high-tech in continuous application and exploration, and to acquire the mathematical knowledge and mathematical qualities required by modern science and technology: to use modern educational technology to demonstrate the mathematics teaching process. Verification experiments, through mathematical calculations, mathematical experiments of symbol processing, introduce mathematics applications into theoretical teaching, and improve students' awareness of the application of mathematics.

In order to adapt to the requirements of strengthening students' mathematics quality and ability assessment, and to cooperate with the reform of mathematics teaching content and teaching methods in higher vocational schools, we can reform the assessment method of mathematics: divide the students' overall evaluation results into three parts: one is the usual score (accounting for 30\%), including daily homework, questioning, class speech, mathematics test results, etc.; second, open test scores (30\%), this part of the assessment is carried out in mathematical modeling, freely combined by students, several people Group, teachers design well in advance (such as measurement, data analysis, statistics, etc.), specify the deadline for completion, students can find relevant information according to needs, and analyze the results of the calculation, combined with the actual feasibility It is recommended that the score be submitted in the form of a paper or an experimental report. The third is the closed-book test score (40\%). This part is based on the assessment of the basic concepts and basic computing skills of students. It is completed in a limited time according to the traditional test method.

\section{Conclusion}

Mathematical teaching can only be rewarded if the teacher is fully engaged. Otherwise, he can only walk in the form. The reform of mathematics teaching in higher vocational colleges requires a long-term adherence and long-term efforts. The reform of mathematics teaching has a long way to go. It is far from enough to rely on the efforts of some teachers. It must be supported by the school, the participation of students, and the active cooperation of teachers. And inter-school collaboration.

\section{References}

[1] Zhan Desheng. Reflections on Higher Mathematics Teaching [J]. Vocational Education Research, 2007.

[2] Liu Runhui. Reflections on Higher Mathematics Teaching in Vocational Colleges [J]. Vocational Education Research, 2006.

[3] Ni Man. Positioning and Reform of Higher Mathematics in Colleges and Universities [J]. Vocational Education Research, 2007.

[4] Han Dengli, Wang Ling, Yan Dong. Research and Practice on Higher Mathematics Teaching Reform in Colleges and Universities [J]. China Science and Technology Information, 2008.

[5] Xie Xiaxia. Reflections on the Orientation and Reform of Higher Mathematics in Colleges and Universities [J]. China Vocational and Technical Education, 2011. 